\title{
A Wearable Fall Detector for Elderly People
}

\author{
Miroslav Musalek
}

Tomas Bata University in Zlin, Faculty of Applied Informatics, Nad Stranemi 4511, Zlin 760 05, Czech Republic
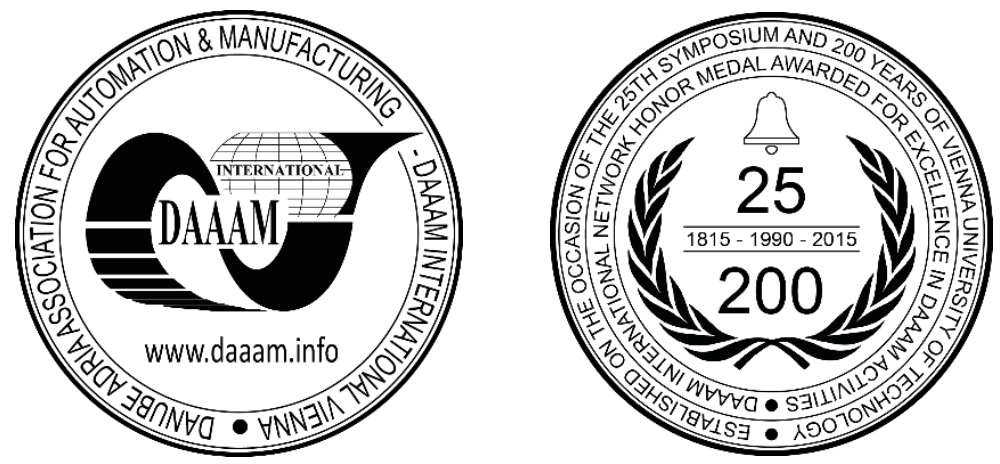

This Publication has to be referred as: Musalek, M[iroslav] (2017). A Wearable Fall Detector for Elderly People, Proceedings of the 28th DAAAM International Symposium, pp.1015-1020, B. Katalinic (Ed.), Published by DAAAM International, ISBN 978-3-902734-11-2, ISSN 1726-9679, Vienna, Austria

DOI: $10.2507 / 28$ th.daaam.proceedings.141

\begin{abstract}
Falls and their subsequent possible side effects are for elderly people and people with various illnesses, such as epilepsy, are present the main obstacle to an independent and more dignified life. Even for people living independently, falls are common occurrences. Proper use of a fall detector will not only add more security to the seniors, but will also allow for timely medical intervention that can be life-critical. One of the limitations of this wearable technology is its limited lifetime and the occurrence of possible errors during the data acquisition and processing process. A fall detector designed to be able to monitor the activity of elderly people and designed to make the necessary warnings to ensure their safety as a result of the fall. In addition, other options that are used for this purpose are compared. This paper summarizes the functionality, architecture and implementation of the system.
\end{abstract}

Keywords: Accelerometer; Gyroscope; Wearable; Fall detection

\section{Introduction}

Falls present a great risk of injury, especially for older people and people suffering from neurodegenerative diseases. Falls and related consequences have various definitions.

A fall can be defined as a change in position that ends with body contact with the ground, which may be accompanied by impaired consciousness and injury. Falls affects $20-30 \%$ of people aged 65-69 years, and up to 50\% of people over 85 years of age in a given calendar year. There is a higher incidence of falls among women, acute and chronically ill patients, hospitalized and long-term institutional care patients [1].

In 1987 the Kellogg international working group on the prevention of falls in the elderly defined and fall as "unintentionally coming to ground, or some lower level not as a consequence of sustaining a violent blow, the loss of academic press journal consciousness, sudden onset of paralysis as in a stroke or an epileptic seizure " [2].

World health organization (WHO) defines a crash as-defined as an event which results in a person coming to rest inadvertently on the ground or floor or other lower level. 
Research on the automatic detections of falls has been taking place since the 1990s. Lord a Colvin [3] studied the causes and consequences of falls in seniors, tried to prevent falls and proposed the use of a fall detection accelerometer. The first prototype fall detector was developed in 1998. There was use a piezoelectric shock sensor to detect abnormal falls and a mercury tilt switch to detect the user's orientation after falling.

Sixsmith et al. [4] in 2004 a range of cheap infrared cameras mounted on the wall was used. The alarm was triggered if activity was not recorded for too long or upon the detection of a fall. The attempts $(20$ falls +10 attempts without falling) unfortunately showed that only $30 \%$ of falls were being correctly determined.

Nyan et al. [5] attempted fall detection based on a high-speed camera and 3 gyroscopes built within an undershirt. They were specifically placed on the chest, armpit and waist. The camera was used to study the body's position during the fall, while the angular velocity was a guide to the fall detection.

Miaou et al. [6] carried out a fall detection based on a panoramic camera and user information (body height to width ratio and BMI. This system has $70 \%$ accuracy without user information and $81 \%$ accuracy with this information.

Bianchia et al. [7] have developed a fall detection system based on a barometric pressure sensor and a triaxial accelerometer located on the waist. Based on the assumption that the atmospheric pressure between the waist and ground level is different, then the results of the experiments demonstrated that the obtained sensor information is useful for the detection of falls.

In recent years, interest in exploring the use of detectors in mobile phones has grown. Other studies [8], however demonstrated that a three-axis accelerometer built into a smartphone may be of relatively low quality and this means worse fall detection results compared to other accelerometers available on the market.

Current work on automatic fall detection methods can be classified into three main categories in terms of the sensors they use: video-based methods, acoustics-based methods and wearable sensor-based methods.

In the article [9] a comparison of the various fall detecting possibilities is performed and we can discover the most suitable detection methods and their effectiveness.

Currently methods using triaxial acceleration sensors are the main methods for motion and fall detection in older populations:

1) $[10]$

2) $[11]$

3) $[12]$

Nyan and colleagues have developed a fall detector using a triaxial accelerometer (MEMS) to collect data. To collect signals use dictated wavelet transformation (DWT). These data are processed to recognize daily activity and fall. The sensor is placed in a jacket at shoulder-height and other components, such as the microcontroller, are inside the pocket. This solution has a limited period of use [13].

Using the accelerometer and the gyroscope together allows for more accurate fall detection. The accelerometer can provide kinetic power while the gyroscope can help estimate the current position.

$\mathrm{Li}$ and colleagues used the accelerometer and gyroscope. By using two triaxial accelerometers located at various points in the body, they can recognize individual daytime activities. Transitions between these static positions are treated as dynamic transitions. If the transition before going to sleep is not intentional, a fall is detected. The recognition of whether movement gradients are intentional or not are determined by measuring linear acceleration and angular velocity [14].

Accelerometer data may sometimes be distorted. Kuncar [15] compares various filtration methods and calibration methods for MEMS sensors in order to remove measurement noise.

In the Czech Republic the Senior Inspect is being implemented, whose development began in 2009 and currently offers continuous assistance in the lives of seniors.

This paper introduces a proposed sensor for detecting the movement of older people.

\section{Fall detection}

The motion sensor is used here this purpose. The sensor is a wearable device capable of wireless communication. This device is capable of recognizing user activity and evaluating falls. Using a triaxial accelerometer, we obtain acceleration along three $\mathrm{X}, \mathrm{Y}$ and $\mathrm{Z}$ coordinate axes. The angular acceleration and orientation of the sensor can be measured using a triaxial gyroscope.

In order to detect falls, the sensor must be able to sense the motion and the various measurable properties associated with motion.

\subsection{Hardware Descriptions}

The proposed fall detection system is shown in Fig. 1. Its individual elements are described in the text below. 


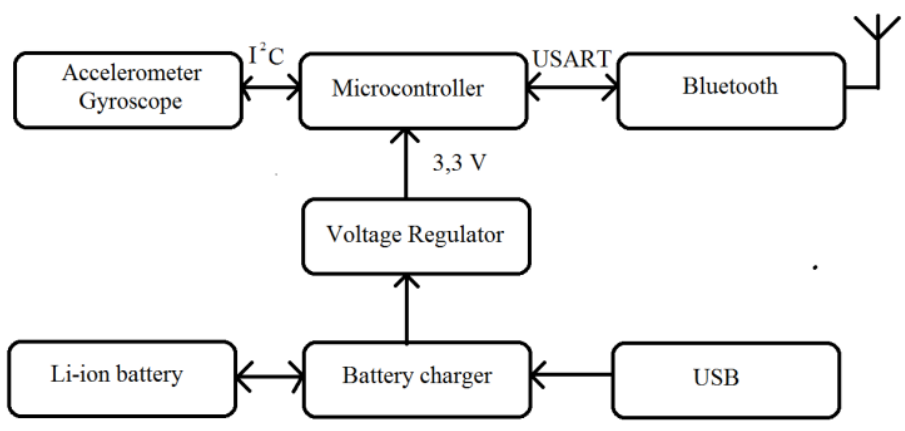

Fig. 1. Block diagram of the proposed fall detection system

I. Motion module

A MEMS sensor MPU-6050, which includes a triaxial accelerometer, a triaxial gyroscope and a thermometer. It also includes the Digital Motion Processor ${ }^{\mathrm{TM}}$ (DMP), which allows the performance of complex calculations between the accelerometer and the gyroscope. For both fast and slow movements, the gyroscope can programmatically set the measuring range to $\pm 250, \pm 500, \pm 1000$ and $\pm 2000^{\circ} / \mathrm{sec}$, and accelerometer within $\pm 2 \mathrm{~g}, \pm 4 \mathrm{~g}, \pm 8 \mathrm{~g}$, and $\pm 16 \mathrm{~g}$. The sensor operates at a supply voltage range of $2.375 \mathrm{~V}$ to $3.46 \mathrm{~V}$. In normal operation, the consumption of the gyroscope, accelerometer, and DMP is $3.9 \mathrm{~mA}$. Due to its small size and low power consumption it is suitable for wearable devices. Its power is selected at $3.3 \mathrm{~V}$.

II. Interface for communication

Wireless detector communication uses the Bluetooth $₫$ standard. For the largest range of equipment, which is $100 \mathrm{~m}$, the Bluetooth $® R N 41$ Class 1 is used. This module includes a built-in antenna. Communication takes place asynchronously through the USART interface (Universal Synchronous/Asynchronous Receiver and Transmitter).

III. Microcontroller

As the microcontroller for the detector, the STM32F051x model is used, which has a high performance ARM Cortex TM -M0 32-bit RISC core operating at $48 \mathrm{MHz}$ Its advantages include low consumption, dimensions and I2C and UART bus. The flash memory size of this model is $64 \mathrm{kB}$. The motion sensor is connected to it through the $\mathrm{I} 2 \mathrm{C}$ bus.

\section{Detector power module}

The power block of the human motion detection module is composed of a battery, a battery charging circuit, and a power circuit. The battery in the module can be charged through the USB interface.

A lithium ionic battery LP-443440-IS-3 is used at a capacity of $600 \mathrm{mAh}$ at $0.2{ }^{\circ} \mathrm{C}$ and nominal voltage of $3.7 \mathrm{~V}$ The capacity of the battery will last for 24-hour operation. The maximum charging current is $560 \mathrm{~mA}$ and the charging voltage is $4.2 \mathrm{~V}$. That is why the BQ24032A circuit from Texas Instruments was chosen as the integrated charging circuit for this battery through the USB interface. This circuit can be used to charge single-cell Li-Ion or Li-Pol batteries. This is a linear charger with an energy consumption management system designed for portable applications.

The MPU-6050 can be powered in the range 2,375 - 3,46 V, Bluetooth ${ }^{\circledR}$ module 3 - $3.3 \mathrm{~V}$ and microcontroller 2 - 3.6 $\mathrm{V}$. Out of the voltage ranges, a uniform voltage of $3.3 \mathrm{~V}$ was chosen. To maintain a constant voltage, the step-up and step-down of the inverter must be combined because the voltage from the BQ24032A circuit output may be below the 3.3V threshold. These requirements are met by the TPS61201 circuit designed to power the circuitry from the battery. In order to maintain the output voltage of $3.3 \mathrm{~V}$, the input voltage must be within the range of $0.3-5.5 \mathrm{~V}$. The converter is based on a fixed frequency, a pulse (PWM) with the aid of synchronous repairs to achieve maximum efficiency. During low load currents, it enters standby mode and maintains high circuit efficiency

\subsection{Fall detection algorithm}

To determine the overall acceleration vector and angular velocity, similar parameters are used as in the [16] study. The total acceleration vector we can call the vector size signal (SVM) can be calculated from the relationship (1).

$$
A c C=\sqrt{\left(A C C_{x}\right)^{2}+\left(A C C_{y}\right)^{2}+\left(A C C_{z}\right)^{2}}
$$

The Accx, Accy, and Accz constants are gravitational accelerations in the $\mathrm{x}, \mathrm{y}$ and accelerometer axes $(\mathrm{g})$.

The equation (2) is designed to calculate the angular velocity of the connected gyroscope. 


$$
\text { Gyro }=\sqrt{\left(\text { Gyro }_{x}\right)^{2}+\left(\text { Gyro }_{y}\right)^{2}+\left(\text { Gyro }_{z}\right)^{2}}
$$

In the upper equation we mean constants Gyrox, Gyro y and Gyro $\mathrm{z}$ angular velocity in the $\mathrm{x}, \mathrm{y}$ and $\mathrm{z}$ axes.

To determine a fall, thresholds are set to make it possible to recognize the fall. This algorithm not only detects the fall, but also compares boundary values with daily activity. Daytime activity includes, in particular, normal walking or running. Prior to evaluating the detection, the potential fall is evaluated by a potential fall limit, which was determined by gradual measurement to $1 \mathrm{~g}$ (picture below). After this evaluation, it will check for orientation changes. If a change in orientation occurs, the fall information is sent. The course of the entire algorithm is represented by Fig. 2.

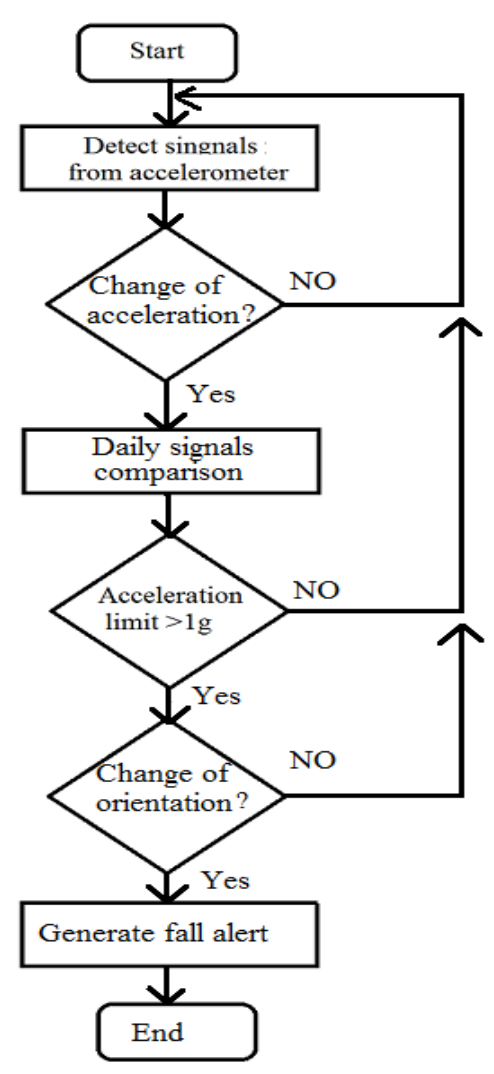

Fig. 2. Fall detection algorithm

Daily activity is determined by the diameter of the acceleration vector and the standard deviation. Values were determined by gradual measurement and effectively set as accurately as possible to identify daily movements such as running and walking. Using a gyroscope, we are able to determine the person's orientation, for example, whether it lies on the right or left side.

Furthermore, sensitivity and specificity measurements were performed to determine the reliability of this system. The above parameters can be calculated from equations (3) and (4).

$$
\begin{aligned}
& \text { Sensitivity }=\frac{T P}{T P+F N} \\
& \text { Specificity }=\frac{T N}{T N+F P}
\end{aligned}
$$

True Positive (TP) is a status where the system can recognize the fall, when it actually occurred. False Positive (FP) is a status where the system detects a fall, although it did not occur. True Negative (TN) means a status in which the system does not detect a fall and a fall actually did not occur. False Negative (FN) is a system state where you cannot detect a fall when it occurs.

In Fig. 3, we see the dependency of the acceleration vector size over time. From the measured data, an accelerometer with a range of at least $\pm 4 \mathrm{~g}$ is required to detect falling, walking and running. The idle state of the sensor corresponds to an acceleration of approximately $1 \mathrm{~g}$, walking to $2.5 \mathrm{~g}$ and running $2.5 \mathrm{~g}$ or more. 


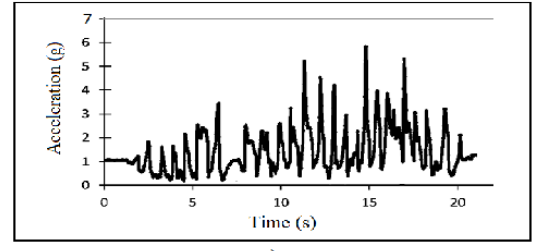

a)

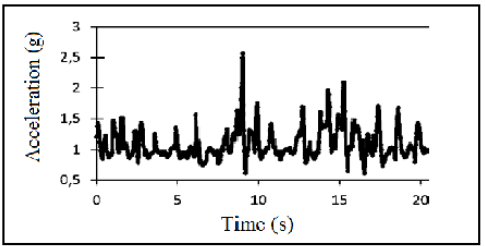

b)

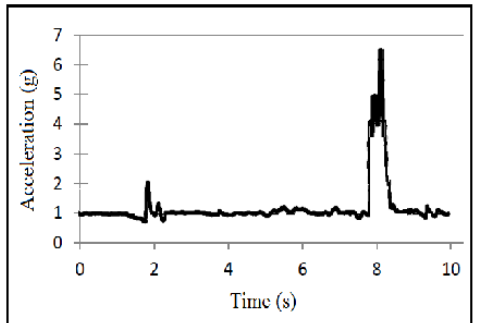

c)

Fig. 3. Acceleration data a) running, b) walking and c) fall detection

The size of the acceleration of the fall ranges from $2 \mathrm{~g}$ and more. Fall detection only with maximum acceleration and subsequent idling is not possible since erroneous detection can occur with faster movement and stopping, as e.g. referred walking or running. To eliminate false detection, it is possible to evaluate the fall warning only when the orientation of the sensor is changed.

\section{Conclusion and future work}

In this article, a fall detector is designed and implemented. Fall detection is an important part in the care of the older population. Because falls are often underestimated, although they significantly complicate life. They often may lead to the lowering of the body's capabilities, such as disability and the impaired quality of life. These factors, the loss of certainty or fear can cause other disorders. For example, depression, powerlessness and social isolation. These measurements were performed with a lateral detector. The acceleration and angular velocity data obtained from the sensors was evaluated by the relevant algorithm. The most important function is to detect a fall that is evaluated when the acceleration greater than $1 \mathrm{~g}$. Suppressing false falls is addressed by detecting daily activity and changing orientation. Measurement results demonstrate that this system can detect the sensitivity and specificity of $92.3 \%$ and $87.6 \%$ of each other.

For the recognition of either standing or sitting, it would be best to use on your thigh, but then it would prevent the detection of lying down. When using with hands, it is not possible to precisely determine the position of the measured object. In case of chest or hand use, the module could be equipped with a heart rate sensor. Further examination will have a physiological effect on the accuracy of the measurement itself. Furthermore, the system can be equipped with a GSM and GPS module.

In the future, portable sensors (e.g. body temperature, glucose, blood pressure, etc.) may be integrated into this system in order to ensure full health care. The fall detection algorithm itself can be adapted to detect traffic accidents that mathematically correspond to the energy impact of a fall. It is further necessary to distinguish between laboratory measurements that may not always correspond to reality. In the foreseeable future, it was necessary to create an extensive database for this purpose. However, this procedure may impact the area of the individual's privacy.

\section{Acknowledgments}

This work was supported by the European Regional Development Fund under the project CEBIA-Tech Instrumentation No. CZ.1.05/2.1.00/19.0376 and also by the Internal Grant Agency of Tomas Bata University under the project No. IGA/FAI/2017/015.

\section{References}

[1] Topinkova, E. (2005). Geriatrics for practice, Prague, ISBN 80-7262-365-6

[2] Gibson, M.J. et al. (1987). "The prevention of falls in later life. And report of the Kellogg International Work Group on the Prevention of Falls by the Elderly "Danish Medical Bulletin, Supplement 34 4:1-24, 1987.

[3] Brownsell, S. a M. S. Hawley (2004). Automatic fall detectors and the fear of falling. Journal of Telemedicine and Telecare of. 10 (5): 262-266 DOI: 10.1258/1357633042026251. ISSN 1357-633x.

[4] Pannurat, Natthapon, Surapa Thiemjarus a Ekawit Nantajeewarawat (2014). Automatic Fall Monitoring: A Review. Sensors. 14(7): 12900-12936. DOI:10.3390/s140712900. ISSN 1424-8220.

[5] Annual International Conference of the IEEE Engineering in Medicine and Biology Society: Lyon, France, 22-26 August 2007. 2007. Piscataway, NJ: IEEE Service Center [distributor], 16 v. (cx, 6759, 13, 46 p.). ISBN 14-2440787-7. 
[6] Miaou, S.-G., Pei-hsu Sung a Chia-Yuan Huang (2006). A Customized Human Fall Detection System Using OmniCamera Images and Personal Information. In: 1st Transdisciplinary Conference on Distributed Diagnosis and Home Healthcare, 2006. D2H2. IEEE, s. 39-42. DOI: 10.1109/DDHH.2006.1624792. ISBN 1-4244-0058-9.

[7] Shane Kim, Jihoon Ryoo (2017). "WiSDom: A model-driven solitary death prevention system based on WiFi signals and real-time supervised training", Consumer Communications \& Networking Conference (CCNC) 2017 14th IEEE Annual, pp. 131-136, 2017, ISSN 2331-9860

[8] Albert MV, Kording K, Herrmann M, Jayaraman A (2012). Fall Classification by Machine Learning Using Mobile Phones. PLoS ONE 7 (5): e36556. https://doi.org/10.1371/journal.pone.0036556

[9] Igual, Raul, Carlos Medrano and Inmaculada Plaza (2013). Challenges, issues and trends in fall detection systems. BioMedical Engineering OnLine. 12(1): DOI: 10.1186/1475-925X-12-66. ISSN 1475-925 x.

[10] Sriborrirux, W.; Leamsumran, P.; Dan-klang,P. (2014). Real-time system for monitoring activity among the elderly using an RF SoC device with triaxial accelerometer data over a wireless sensor network:2014 IEEE MTT-S International Microwave Workshop Series on RF and Wireless Technologies for Biomedical and Healthcare Applications(IMWSBio), Thailand,December 8-10,2014 [C].IEEE,2014.

[11] Yanjun Li,Gan Chen,Yueyun Shen,et al. (2012). Accelerometer- based fall detection sensor system for the elderly:2012 IEEE 2nd International Conference on Cloud Computer and Intelligent Systems (CCIS),Hangzhou, China, October 30- November 1,2012[C].IEEE,2012.

[12] Aguiar,B.;Rocha,T.;Silva,J., et al. (2014). Accelerometer- based fall detection for smartphones: 2014 IEEE International Symposium on Medical Measurements and Applications (MeMeA), June 11-12, 2014 [C].IEEE,2014.

[13] Nyan, M., et al. (2006). Garment-based detection of falls and activities of daily living using 3-axis MEMS accelerometer. in Journal of Physics: Conference Series. 2006. IOP Publishing.

[14] Li, Q., et al. (2009). Accurate, fast fall detection using gyroscopes and accelerometer-derived posture information. in Wearable and Implantable Body Sensor Networks, 2009. BSN 2009. Sixth International Workshop on. 2009. IEEE.

[15] Kuncar, A[les] (2016). Basic Techniques for Filtering Noise Out of Accelerometer Data, Proceedings of the 26th DAAAM International Symposium, pp.1122-1128, B. Katalinic (Ed.), Published by DAAAM International, ISBN 978-3-902734-07-5, ISSN 1726-9679, Vienna, Austria DOI: 10.2507/26th.daaam.proceedings.158

[16] Quoc T. Huynh, Uyen D. Nguyen, Lucia B. Irazabal, Nazanin Ghassemian, and Binh Q. Tran (2015). “Optimization of an Accelerometer and Gyroscope-Based Fall Detection Algorithm," Journal of Sensors, vol. 2015, Article ID 452078, 8 pages, 2015. doi:10.1155/2015/452078 